\title{
BMJ Open Gastric emergencies in older adults: not always the same old story! A tertiary care emergency department observational cohort study
}

\author{
Fausto Rosa (D) , ${ }^{1}$ Marcello Covino, ${ }^{2}$ Pietro Fransvea, ${ }^{3}$ Valerio Cozza, ${ }^{3}$ \\ Giuseppe Quero, ${ }^{1}$ Claudio Fiorillo, ${ }^{1}$ Benedetta Simeoni, ${ }^{2}$ Antonio La Greca, ${ }^{3}$ \\ Gabriele Sganga, ${ }^{3}$ Antonio Gasbarrini, ${ }^{4}$ Francesco Franceschi, ${ }^{2}$ \\ Guido Costamagna, ${ }^{5}$ Sergio Alfieri ${ }^{1}$
}

To cite: Rosa F, Covino M, Fransvea P, et al. Gastric emergencies in older adults: not always the same old story! A tertiary care emergency department observational cohort study. BMJ Open 2022;12:e056981. doi:10.1136/ bmjopen-2021-056981

- Prepublication history for this paper is available online. To view these files, please visit the journal online (http://dx.doi. org/10.1136/bmjopen-2021056981).

FR and MC contributed equally.

Received 31 August 2021 Accepted 29 November 2021

Check for updates

(C) Author(s) (or their employer(s)) 2022. Re-use permitted under CC BY-NC. No commercial re-use. See rights and permissions. Published by BMJ.

For numbered affiliations see end of article.

Correspondence to

Dr Fausto Rosa;

fausto.rosa@policlinicogemelli.it

\section{ABSTRACT}

Objective To analyse and compare the clinical outcomes between patients $\geq 80$ years and $65-79$ years, who presented to our emergency department (ED) with the two most common gastric emergency (GE) clinical presentation, that is, gastric bleeding and gastric perforation.

Design Single-centre retrospective cohort study. Participants A total of 1011 patients were enrolled: 421 patients aged $\geq 80$ years were compared with a group of 590 patients aged 65-79 years.

Primary and secondary outcome measures The primary outcome was to compare the overall mortality. Secondary outcomes included major complications, in-hospital length of stay (LOS) and need for surgical procedures.

Results Patients $\geq 80$ years with GE had different presentations at ED compared with younger patients: they had higher gastrointestinal bleeding $(24.9 \%$ vs $16.3 \%$, $\mathrm{p}=0.001)$, anemisation $(14.5 \%$ vs $8.8 \%, \mathrm{p}=0.005)$ and shock $(10.9 \%$ vs $5.1 \%, p=0.001)$ rates. Median LOS, cumulative major complications and mortality rates were similar among the two groups.

Multivariate analysis identified shock, gastric malignancy and gastric fistula as independent predictors for survival. Conclusions Patients $\geq 80$ years with GE did not have a higher mortality rate and cumulative major complications as compared to younger elderly patients. Invasive treatments were not associated with a different prognosis in this age group.

\section{INTRODUCTION}

Gastric emergencies (GEs) include a wide range of conditions of variable severity.

Clinical presentation may significantly differ from a sudden event, such as an acute gastric bleeding or perforation, to more chronic but potentially fatal conditions. The correct approach to these clinical scenarios is mandatory to prevent or minimise the risk of poor prognosis. ${ }^{1}$

\section{Strengths and limitations of this study}

- A single-centre, retrospective observational cohort study was conducted in elderly patients with the two most common gastric emergencies (GEs), that is, gastric bleeding and gastric perforation.

- This study is strengthened by its sample size, representing the largest experience in elderly patients with GE.

- Potential biases are strictly related to its retrospective nature.

- Its single-centre design does not represent all patients admitted with a GE in a general emergency department.

Adequate management of such disorders is complex and inevitably multidisciplinary, demanding a prompt diagnosis and therapeutic procedures that may involve internists, gastroenterologists, radiologists, intensive care physicians and surgeons.

Knowledge of the mechanisms underlying these pathologies is of crucial importance to guarantee an adequate approach of severe gastrointestinal emergencies, requiring a wider awareness from the physicians involved. Moreover, endoscopy represents a supporting pillar in both the diagnostic and, more importantly, minimally invasive and effective therapeutic interventions. ${ }^{1}$

Therefore, considering the increasing incidence of GE and the contemporary and progressive ageing of the population, it is becoming of extreme importance to define. Among this heterogeneous cohort, it is important to analyse the clinical outcomes according to the different age subsets within the geriatric population with particular regard to oldest old patients. 
The aim of this study was to analyse and compare the clinical outcomes between patients $\geq 80$ years and $65-79$ years, who presented to our emergency department (ED) with the two most common GEs, that is, gastric bleeding and gastric perforation.

\section{METHODS}

\section{Study design}

This is a single-centre, retrospective study conducted at Fondazione Policlinico Universitario A. Gemelli IRCCS in Rome, Italy, where the ED has annual attendance at the ED of about 75000 patients (more than $87 \%$ adults). After approval by our institutional review board, all the clinical records of consecutive patients admitted to our ED from 1 January 2014 to 31 December 2018 were evaluated and patients presenting GE in this period were recruited.

All patients with a diagnosis of GE (gastric bleeding or gastric perforation) (International Classification of Disease, Ninth Revision, Clinical Modification (ICD9-CM) ICD-9-CM codes 578.0, 531.1, 531.2, 531.4, 531.5, 531.6, 534.01, 534.10, 534.11, 534.20, 534.41, 534.50, $534.51,534.60,534.61,534.71,537.6,537.8,537.83$, 537.84 and 863.1), either as primary diagnosis or as secondary diagnosis but with a complication of gastric cancer, gastric peptic ulcer or gastric angiodysplasia as a primary diagnosis, were recruited for this study.

Patients under 65 years of age were excluded.

All patients were then divided according to age. The oldest old group consisted of patients 80 years of age and older, while those aged 65-79 years represented the control group.

\section{Data collection}

The following demographic and clinical data were collected: age, gender, clinical history (including Charlson Comorbidity Index, proton pump inhibitor (PPI) therapy, acetylsalicylic acid (ASA) therapy, anticoagulant therapy, shock at ED presentation, hypertension, congestive heart failure, peripheral vascular disease, dementia, chronic obstructive pulmonary disease, diabetes and chronic kidney disease), abdominal complications (acute gastritis, gastric ulcer, gastric malignancy, gastric angiodysplasia, gastric perforation and gastric fistula) and treatment (conservative management, minor surgery and major surgery).

Any interventional procedure, complications and perioperative outcomes were registered.

\section{Diagnosis}

GE diagnosis was initially made clinically, according to patients' clinical presentation, later confirmed through a CT scan or an oesophagogastroduodenoscopy.

GE-related specific conditions at presentation, causing bleeding or perforation, included

- Acute gastritis, defined as an inflammation of the gastric mucosa.
- Gastric ulcer, defined as a break in the gastric mucosa.

- Gastric malignancy.

- Gastric angiodysplasia, defined as a small vascular malformation of the stomach.

- Gastric fistula, defined as an abnormal connection between the stomach and the skin.

- Gastric perforation, defined as an abnormal connection between the stomach and the peritoneum.

\section{Clinical management}

In case of gastric bleeding, after patient resuscitation in case of acute clinical presentation, upper gastrointestinal endoscopy was performed as soon as possible, ${ }^{2-4}$ especially in high-risk patients. Endoscopic therapy consisted of either injection or thermal or mechanical methods. In case of endoscopic failure, in selected cases, a transcatheter arterial embolisation was attempted. Surgical intervention was reserved when endoscopic and/or endovascular therapies failed and when perforation or malignancy coexisted. High-dose intravenous PPI was always associated with all procedures.

In case of gastric perforation, most patients were treated by an urgent operation, but there was the possibility for a conservative management. A non-operative management (NOM) was essentially reserved for either asymptomatic or compromised patients.

As 'minor surgery', a percutaneous drainage and/ or laparoscopic peritoneal lavage and drainages were considered.

As 'major surgery' any gastric resection was considered.

\section{Study outcomes}

Mortality was defined as death occurrence in the ED or during ward admission. In-hospital length of stay (LOS) was calculated from ED admission to discharge/death.

Necessity of mechanical ventilation was defined as need for mechanical ventilation in any phase of the hospital stay (apart from eventual major surgery intervention).

Cumulative major complications considered were death, admission to the intensive care unit or need of mechanical ventilation, progression to sepsis and septic shock; sepsis was defined according to Sepsis-3. ${ }^{5}$

\section{Statistical analysis and sample size}

Categorical variables are presented as numbers and percentages, and statistically compared at univariate analysis by $\chi^{2}$ test, with Yates correction if appropriate. Continuous variables are presented as median (quartiles) and compared by the Mann-Whitney U test. A p value of $\leq 0.05$ was considered statistically significant.

Variables significant at univariate analysis were entered into a logistic regression model to identify independent predictor of death or major complications. Logistic regression results are expressed as OR with 95\% CI. All data were analysed by SPSS V.25. 
Patient and public involvement statement

We used administrative data and health check-up data, and hence, patients were not directly involved in this study.

\section{RESULTS}

During the study period, a total of 1011 patients $\geq 65$ years (514 men and 497 women with a median age of 78 (72-83) years) were admitted to our ED with a diagnosis of GE. In this cohort of patients, we evaluated 421 $(41.6 \%)$ patients aged $\geq 80$ years and $590(58.4 \%)$ patients aged $65-79$ years.
Demographic characteristics of the patients included in the study population are shown in table 1 .

As expected, considering the clinical history, Charlson Comorbidity Index, patients on PPI and ASA therapy, hypertension, congestive heart failure, dementia and chronic kidney disease were significantly more frequent in patients $\geq 80$ years (table 1 ).

As far as GE-related specific conditions are concerned, gastric ulcer, gastric fistula and gastric perforation were significantly higher in patients $\geq 80$ years $(\mathrm{p}<0.001)$, whereas only gastric malignancy was significantly higher in patients aged 65-79 years $(\mathrm{p}<0.001)$.

Table 1 Demographic characteristics of the patients in the study population

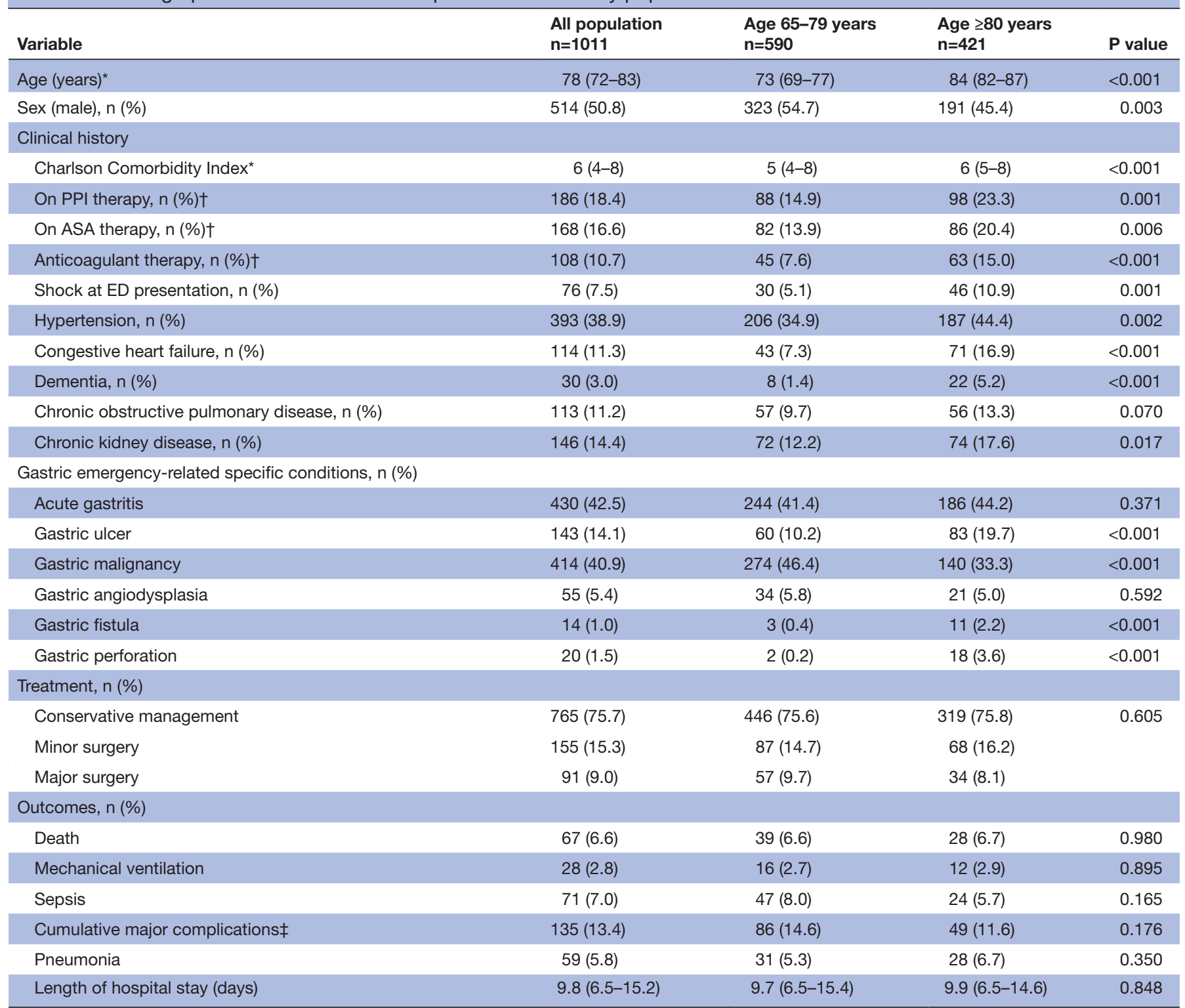

Vital parameters and laboratory values were acquired at ED admission visit. Continuous variables are compared by Mann-Whitney U test. Categorical variables are compared by $\chi^{2}$ test.

*Values are expressed as median (IQR).

†Therapy was defined as continuous assumption in the month before ED access.

$\ddagger$ Cumulative major complications include death, admission to intensive care unit/ventilation and sepsis.

§Hypotension/shock was defined as systolic blood pressure of $<100 \mathrm{~mm} \mathrm{Hg}$ at ED admission.

ASA, aminosalicylic acid; ED, emergency department; PPI, proton pump inhibitor. 
Table 2 Comparison between conservative and interventional management

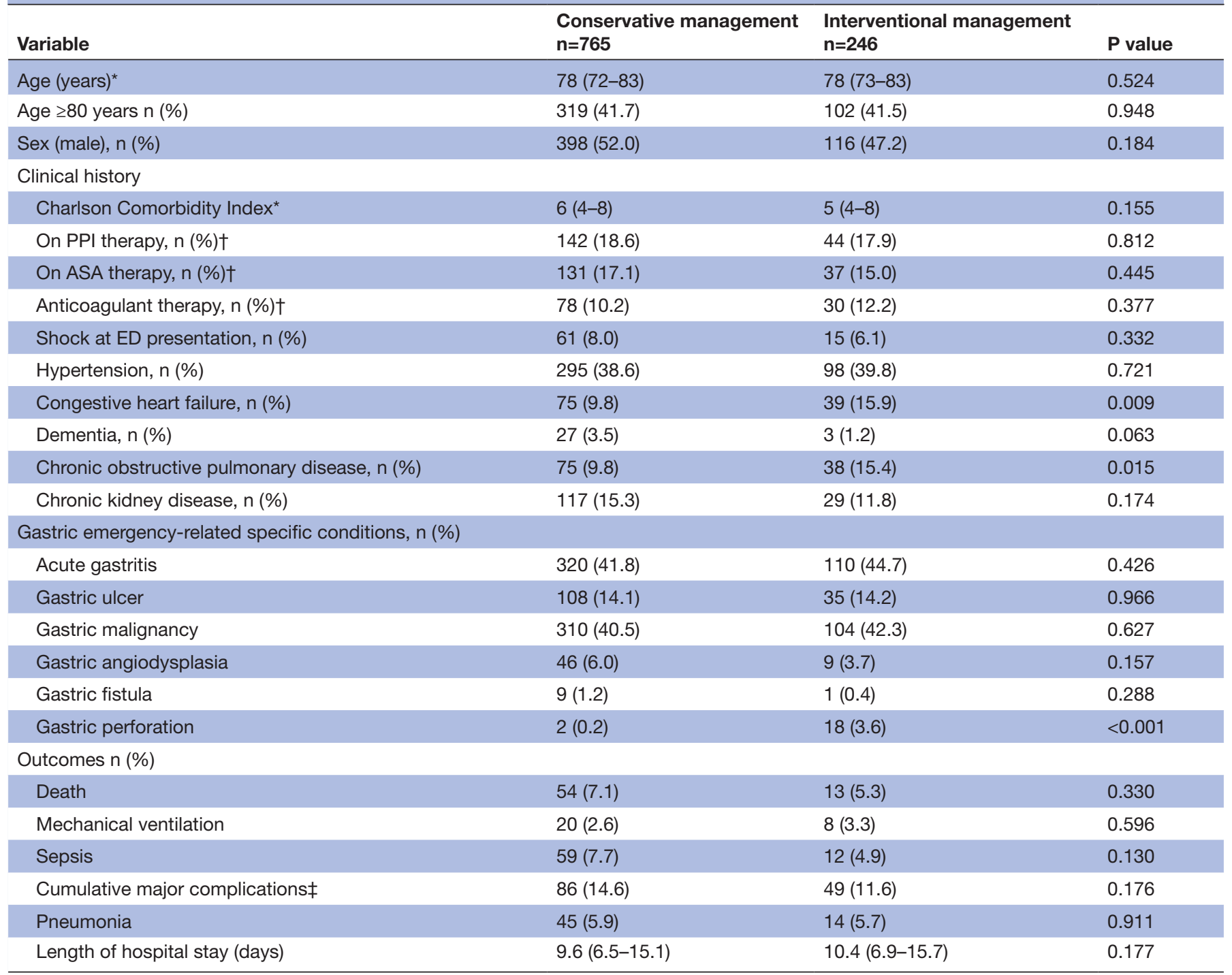

*Values are expressed as median (IQR).

tTherapy was defined as continuous assumption in the month before ED access.

$\ddagger$ Cumulative major complications include: death, admission to intensive care unit/ventilation and sepsis.

$\S$ Hypotension/shock was defined as systolic blood pressure of $<100 \mathrm{~mm} \mathrm{Hg}$ at ED admission.

ASA, aminosalicylic acid; ED, emergency department; PPI, proton pump inhibitor.

No significant difference among the two groups was detected regarding neither treatment nor outcomes.

Comparison between conservative and interventional management is summarised on table 2 .

On the contrary, as far as clinical history is concerned, both congestive heart failure and chronic obstructive pulmonary disease were significantly higher in patients undergoing interventional procedures.

Concerning GE-related specific conditions, as expected, only gastric perforation was significantly higher in patients having an interventional management $(3.6 \%$ vs $0.2 \%, \mathrm{p}<0.001$ ).

No difference was detected regarding outcomes when comparing conservative approach with interventional approach.
The analysis of clinical variables in patients with no complications or minor complications with respect to patients with cumulative major complications is shown on table 3.

Gastric ulcer, gastric malignancy, gastric fistula and gastric perforation were significantly associated with cumulative major complications $(\mathrm{p}=0.003, \mathrm{p}<0.001$, $\mathrm{p}=0.004, \mathrm{p}<0.001, \mathrm{p}=0.033$ and $\mathrm{p}=0.002$, respectively)

On the other hand, hypertension, acute gastritis and gastric angiodysplasia were significantly higher in patients with no complications or minor complications $(\mathrm{p}=0.029$, $\mathrm{p}<0.001$ and $\mathrm{p}=0.024$, respectively).

As expected, LOS was significantly higher in patients with cumulative major complications (15.0 days (9.128.4) vs 9.5 (6.5-13.9), $\mathrm{p}<0.001)$. 
Table 3 Clinical variables in patients with no complications or minor complications, and in patients with cumulative major complications*

\begin{tabular}{|c|c|c|c|}
\hline Variable & $\begin{array}{l}\text { No complications or minor } \\
\text { complications } n=876\end{array}$ & $\begin{array}{l}\text { Cumulative major complications* } \\
\mathrm{n}=135\end{array}$ & $P$ value \\
\hline Age $(\text { years })^{\S}$ & $78(72-83)$ & $77(71-83)$ & 0.317 \\
\hline Age $\geq 80$ years, $n(\%)$ & $372(42.5)$ & 49 (36.3) & 0.176 \\
\hline Sex (male), $\mathrm{n}(\%)$ & $439(50.1)$ & $75(55.6)$ & 0.239 \\
\hline \multicolumn{4}{|l|}{ Clinical history } \\
\hline Charlson Comorbidity Index§ & $6(4-8)$ & $6(5-8)$ & 0.064 \\
\hline On PPI therapy, n (\%)† & $166(18.9)$ & $20(14.8)$ & 0.248 \\
\hline On ASA therapy, n (\%)† & $145(16.6)$ & $23(17.0)$ & 0.888 \\
\hline Anticoagulant therapy, $\mathrm{n}(\%) \dagger$ & $93(10.6)$ & $15(11.1)$ & 0.862 \\
\hline Shock at ED presentation, $\mathrm{n}(\%)$ & $52(5.9)$ & $24(17.8)$ & $<0.001$ \\
\hline Hypertension, n (\%) & $352(40.2)$ & $41(30.4)$ & 0.029 \\
\hline Congestive heart failure, $\mathrm{n}(\%)$ & $99(11.3)$ & $15(11.1)$ & 0.948 \\
\hline Dementia, n (\%) & $25(2.9)$ & $5(3.7)$ & 0.588 \\
\hline Chronic obstructive pulmonary disease, $\mathrm{n}(\%)$ & $99(11.3)$ & $14(10.4)$ & 0.749 \\
\hline Chronic kidney disease, $\mathrm{n}(\%)$ & $123(14.0)$ & $23(17.0)$ & 0.357 \\
\hline \multicolumn{4}{|l|}{ Treatment, $\mathrm{n}(\%)$} \\
\hline Conservative management & $658(75.1)$ & $107(79.3)$ & \\
\hline Minor surgery & $137(15.6)$ & $18(13.3)$ & 0.574 \\
\hline Major surgery & $81(9.2)$ & $10(7.4)$ & \\
\hline \multicolumn{4}{|c|}{ Gastric emergency-related specific conditions, $n(\%)$} \\
\hline Acute gastritis & $401(45.8)$ & $29(21.5)$ & $<0.001$ \\
\hline Gastric ulcer & $113(12.9)$ & $30(22.2)$ & 0.004 \\
\hline Gastric malignancy & $338(38.6)$ & $76(56.3)$ & $<0.001$ \\
\hline Gastric angiodysplasia & $53(6.1)$ & $2(1.5)$ & 0.024 \\
\hline Gastric fistula & $6(0.7)$ & $4(3.0)$ & 0.033 \\
\hline Gastric perforation & $9(1.0)$ & $6(4.4)$ & 0.002 \\
\hline \multicolumn{4}{|l|}{ Outcomes } \\
\hline Length of hospital stay (days) & $9.5(6.5-13.9)$ & $15.0(9.1-28.4)$ & $<0.001$ \\
\hline
\end{tabular}

${ }^{*}$ Cumulative major complications include death, admission to intensive care unit/ventilation and sepsis.

†Therapy was defined as continuous assumption in the month before ED access.

$\ddagger$ Hypotension/shock was defined as systolic blood pressure of $<100 \mathrm{~mm} \mathrm{Hg}$ at ED admission.

$\S$ Values are expressed as median (IQR).

ASA, aminosalicylic acid; ED, emergency department; PPI, proton pump inhibitor.

Table 4 summarises the logistic regression analysis of significant factors at univariate analysis for survival. The multivariate analysis identified gastric malignancy (OR $3.82,95 \%$ CI 1.95 to $7.47 ; \mathrm{p}<0.001)$ and gastric fistula (OR $7.30,95 \%$ CI 2.05 to $26.01 ; \mathrm{p}=0.002$ ) as negative factors associated with survival.

\section{DISCUSSION}

GEs are frequent and dramatic events with both a high mortality rate and a significant cost for healthcare systems worldwide. It has been well demonstrated that older age is an independent prognostic factor for GE, with the risk increasing significantly in individuals aged $>65$ years and increasing further in those aged $>75$ years. ${ }^{6}$
To the best of our knowledge, our study represents the largest series of comparison of clinical outcomes between patients aged $65-79$ years and those $\geq 80$ years presenting with GE.

In the very next future, patients older than 80 years will represent the highest percentage of the elderly patients admitted to an ED. ${ }^{7}$ This population often presents specific clinical conditions compared with younger cohorts. $^{8}$

We particularly analysed the clinical differences strictly related to age and the role of age as an independent prognostic factor for the main clinical outcomes.

According to the most recent pieces of evidence, bleeding incidence and mortality are distinctly higher in 
Table 4 Factors associated with survival at univariate and multivariate analyses (logistic regression model)

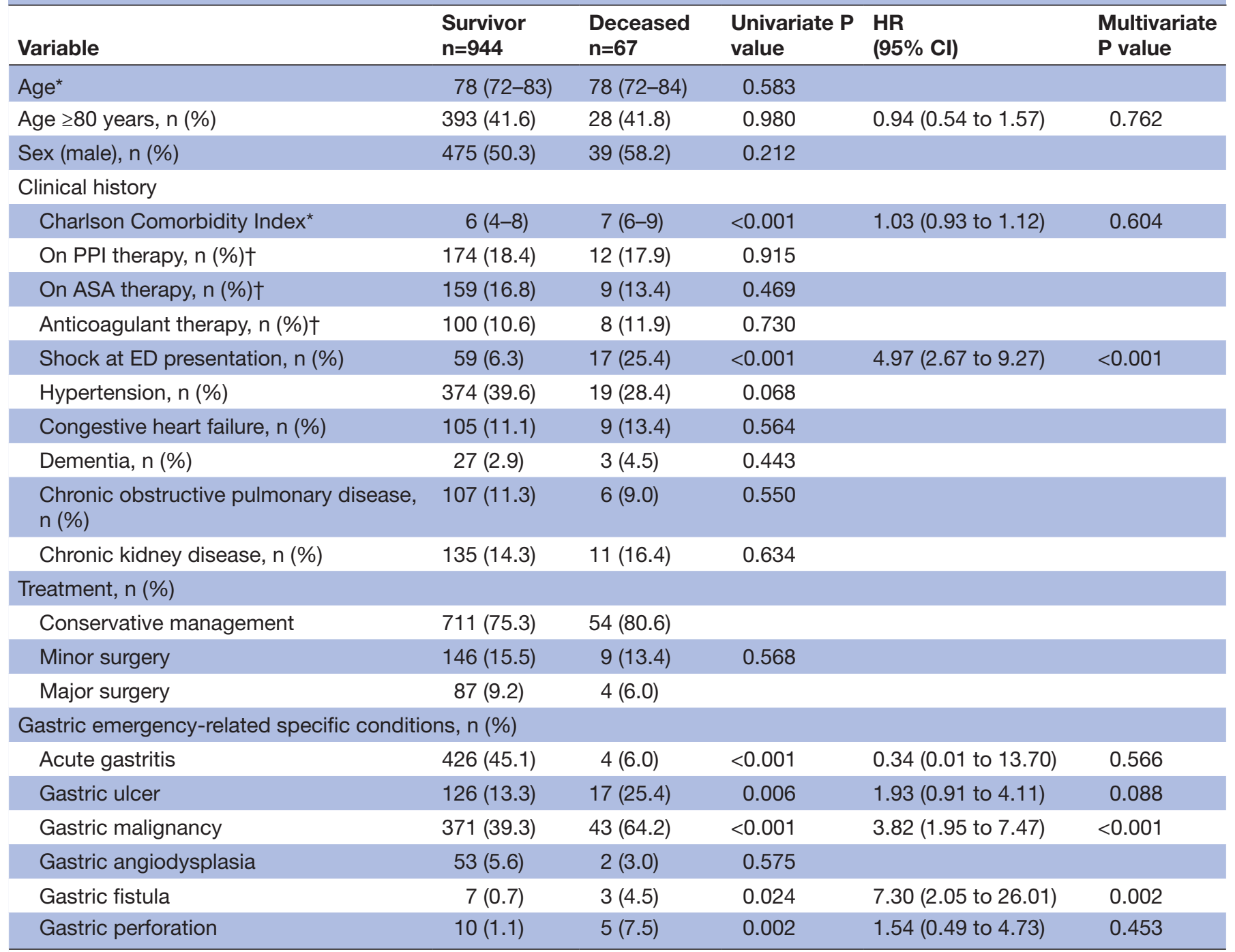

*Values are expressed as median (IQR).

†Therapy was defined as continuous assumption in the month before ED access.

†Hypotension/shock was defined as systolic blood pressure of $<100 \mathrm{~mm} \mathrm{Hg}$ at ED admission.

$\S$ Cumulative major complications include death, admission to intensive care unit/ventilation and sepsis.

ASA, aminosalicylic acid; ED, emergency department; PPI, proton pump inhibitor.

elderly patients, especially in those with comorbidities, with respect to younger ones. ${ }^{6}$ Similarly, gastric perforations occur most often in elderly patients with coexistent medical problems, and these patients are also at increased risk of postoperative morbidity. ${ }^{9}$ Mortality is threefold to fourfold higher in the elderly (up to 50\%) due to occurrence of concomitant medical diseases and the difficulty in differential diagnosis resulting in delayed treatment. $^{1011}$

Non-steroidal anti-inflammatory drug (NSAIDs) therapy and Helicobacter pylori infection are the most frequent triggers involved in acute gastric bleeding and perforation. ${ }^{12}$ The risk of bleeding seems to be higher for NSAID-elated than for $H$. pylori-related ulcers due to the direct antiplatelet action of NSAIDs on the clotting process. ${ }^{13}$
Our data revealed that Charlson Comorbidity Index, PPI therapy, ASA therapy and anticoagulant therapy were significantly more frequent in the oldest old group. However, only Charlson Comorbidity Index had a negative prognostic association with survival, although not confirmed at multivariate analysis.

Moreover, as clearly demonstrated by our data, both gastric bleeding and gastric perforation were significantly more frequent in older patients. However, very interestingly, and in contrast with previously reported data, ${ }^{9-11}$ in our study, the oldest old patients presented similar results in terms of morbidity, mortality and LOS compared with the elderly group.

The prevention of gastric bleeding is crucial, above all for high-risk patients. Unfortunately, for older ones, it is 
quite difficult to follow the several guidelines presented on this topic. ${ }^{14}$

H. pylori eradication is mandatory in all patients with peptic ulcer and is frequently achieved with sequential therapy in elderly patients. ${ }^{12}$

As far as treatment for gastric bleeding in the elderly is concerned, initial evaluation and risk stratification are crucial in the management of these patients. ${ }^{15}$ In elderly patients with gastric bleeding and who are haemodynamically unstable, intensive monitoring and prompt fluid and red cell replacement are mandatory. ${ }^{15}$ Nevertheless, we should always remember that unnecessary transfusion in haemodynamically stable patients was reported to be associated with higher rates of recurrent bleeding and death. ${ }^{15-18}$

Further management requires endoscopy as a first-line treatment (strong recommendation based on low-quality evidences, $1 \mathrm{C}) .{ }^{19}$

After bleeding is controlled by endoscopy, patients with peptic ulcer should be treated with PPI for 6-8 weeks. ${ }^{19}$ Long-term PPI is not recommended unless the patient has ongoing NSAID use (strong recommendation based on moderate-quality evidence, 1B). ${ }^{19}$

Surgery is necessary when endoscopic/angiographic treatments have failed and there is evidence of active bleeding. Moreover, refractory bleeding gastric ulcers should be surgically resected for the elevated likelihood of malignant origin. ${ }^{19}$

In conclusion, acute gastric bleeding is a dramatic event with a high mortality rate, particularly in elderly patients where, as demonstrated by our data, shock represents the most negative prognostic factor.

Moreover, in contrast with the majority of the literature where PPI therapy was considered a preventative strategy and ASA and anticoagulant therapy were risk factors for bleeding and survival, according to our data, neither PPI nor ASA and anticoagulant therapy were prognostically significant.

Regarding acute gastric perforation, the number of elderly patients requiring emergency procedures has increased dramatically. ${ }^{20}$

When associated with peritonitis and sepsis/septic shock, it is a medical/surgical emergency requiring rapid evaluation and management. ${ }^{21}$

NOM of a gastric perforation generally includes nil by mouth, intravenous hydration, decompression via nasogastric tube, antisecretory, PPI therapy and intravenous antibiotics. It represents an attractive management as it prevents surgery and resultant morbidity. The rationale of a NOM is that, in case of small perforations and in the absence of a diffuse peritonitis, the stomach heals spontaneously through omental adhesions, without the need of a surgical procedure..$^{22} 23$

Even if our study represents the largest experience demonstrating similar outcomes between conservative versus interventional approaches in GE in elderly patients, the feasibility of a NOM should be carefully evaluated, always taking into account that an increase in surgical delay significantly impairs surgical outcomes.
An observational Danish study ${ }^{24}$ showed that, over the first 24 hours after ED admission, each hour of surgical delay beyond hospital admission was associated with an adjusted $2.4 \%$ decreased probability of survival with respect to the previous hour. With respect to our data, these authors conclude that elderly patients may experience paradoxical higher mortality if NOM fails, and caution is advised in patients $>70$ years of age. ${ }^{24}$

Moreover, a systematic review ${ }^{25}$ performed in 2010 including 50 studies for a total of 29782 patients demonstrated a clear association of older age, comorbidity and use of NSAIDs with mortality; moreover, shock at admission, preoperative metabolic acidosis, tachycardia, acute renal failure, low serum albumin level, high ASA score and preoperative delay of $>24$ hours were also associated with poor prognosis.

In a recent Italian multicentre prospective study, Fransvea $e t a l^{20}$ demonstrated that laparoscopic gastric repair is safe and feasible in elderly patients with perforation from gastric ulcer and is associated with better perioperative outcomes. However, patient selection and preoperative frailty evaluation in the elderly population are the key to achieve better outcomes. ${ }^{20}$

In case of large gastric ulcer perforation that raises the suspicion of malignancy, a resection is always recommended also in elderly patients, even with a palliative aim. $^{19}$

According to our data, gastric malignancies were significantly more frequent in the elderly group $(46.4 \%$ vs $33.3 \%, \mathrm{p}<0.001$ ), and only $42.3 \%$ had a surgical procedure within 72 hours from ED admission. Moreover, in accordance with the literature, ${ }^{26}$ we demonstrated that the oncological condition was significantly related both to major complications and represented a negative prognostic factor for survival at univariate and multivariate analyses.

\section{CONCLUSIONS}

GEs are still common worldwide and their incidence pattern is strictly related to the rise of new risk factors, that is, the increasing median age in the population, the increasing incidence of $H$. pylori infection, the extensive use of NSAIDs, and the increase in alcohol and smoking abuse. ${ }^{1}$ Despite the tremendous improvement in preventive therapies, the rate of complications of these events is still high and is burdened by high morbidity and mortality.

As reported by our data, between the oldest old group and the elderly group, no statistical difference was observed neither regarding approaches nor in terms of morbidity and mortality. Therefore, in contrast to other emergencies, ${ }^{2728}$ for GEs, a prompt recognition and treatment of the complications could lead to similar outcomes in both oldest old and elderly patients.

\section{Study limitations}

Despite our study representing the largest experience on GE clinical course in elderly patients, some major 
limitations need to be underlined. First, potential biases are strictly related to its retrospective nature. Second, its single-centre design does not represent all patients admitted with a GE in a general ED.

In any case, our results provide a significant contribution to the current knowledge on GE in geriatric patients, showing that chronological age does not significantly affect neither mortality nor cumulative major complications when patients are promptly and accurately treated.

\section{Author affiliations}

${ }^{1}$ Digestive Surgery, Fondazione Policlinico Universitario Agostino Gemelli IRCCS, Roma, Italy

${ }^{2}$ Emergency Medicine, Fondazione Policlinico Universitario Agostino Gemelli IRCCS, Roma, Italy

${ }^{3}$ Emergency Surgery, Fondazione Policlinico Universitario Agostino Gemelli IRCCS,

Roma, Italy

${ }^{4}$ Internal Medicine, Fondazione Policlinico Universitario Agostino Gemelli IRCCS, Roma, Italy

${ }^{5}$ Digestive Endoscopy, Fondazione Policlinico Universitario Agostino Gemelli IRCCS, Roma, Italy

Contributors Study conception and design: FR, MC, BS, PF, VC, ALG, GS, AG, $\mathrm{FF}, \mathrm{GC}$ and $\mathrm{SA}$. Acquisition of data: MC, FR, BS, GQ, CF, PF, VC and ALG. Analysis and interpretation of data: MC, FR, GQ, CF, GS, AG, FF, GC and SA. Drafting of the manuscript: MC, FR, BS, GQ, CF, PF, VC and ALG. Critical revision: GS, FF, AG, GC and $S A$. FR acts as the guarantor for the work.

Funding The authors have not declared a specific grant for this research from any funding agency in the public, commercial or not-for-profit sectors.

Competing interests None declared.

Patient and public involvement Patients and/or the public were not involved in the design, conduct, reporting or dissemination plans of this research.

Patient consent for publication Consent obtained directly from patient(s).

Ethics approval This study involves human participants and was approved by ethics committee of Catholic University of Rome (\#0032821/20). Participants gave informed consent to participate in the study before taking part.

Provenance and peer review Not commissioned; externally peer reviewed.

Data availability statement All data relevant to the study are included in the article or uploaded as supplementary information. Extra data can be accessed via the Dryad data repository at http://datadryad.org/ with the doi: 10.5061/dryad. h18931znh.

Open access This is an open access article distributed in accordance with the Creative Commons Attribution Non Commercial (CC BY-NC 4.0) license, which permits others to distribute, remix, adapt, build upon this work non-commercially, and license their derivative works on different terms, provided the original work is properly cited, appropriate credit is given, any changes made indicated, and the use is non-commercial. See: http://creativecommons.org/licenses/by-nc/4.0/.

\section{ORCID iD}

Fausto Rosa http://orcid.org/0000-0002-7280-8354

\section{REFERENCES}

1 Dinis-Ribeiro M, Amaro P. Management of gastrointestinal emergencies. Best Pract Res Clin Gastroenterol 2013;27:631-2.

2 Cook DJ, Guyatt GH, Salena BJ, et al. Endoscopic therapy for acute nonvariceal upper gastrointestinal hemorrhage: a meta-analysis. Gastroenterology 1992;102:139-48.

3 Marmo R, Rotondano G, Bianco MA, et al. Outcome of endoscopic treatment for peptic ulcer bleeding: is a second look necessary? A meta-analysis. Gastrointest Endosc 2003;57:62-7.
4 Tsoi KKF, Chiu PWY, Sung JJY. Endoscopy for upper gastrointestinal bleeding: is routine second-look necessary? Nat Rev Gastroenterol Hepatol 2009;6:717-22.

5 Singer M, Deutschman CS, Seymour CW, et al. The third International consensus definitions for sepsis and septic shock (Sepsis-3). JAMA 2016;315:801-10.

6 Zullo A, Hassan C, Campo SMA, et al. Bleeding peptic ulcer in the elderly: risk factors and prevention strategies. Drugs Aging 2007;24:815-28.

7 United Nations DoE, Social Affairs PD. World population ageing 2015 (st/esa/ser. A/390. New York, 2015.

8 Covino M, Petruzziello C, Onder G, et al. A 12-year retrospective analysis of differences between elderly and oldest old patients referred to the emergency department of a large tertiary hospital. Maturitas 2019;120:7-11.

9 Weledji EP. An overview of gastroduodenal perforation. Front Surg 2020;7:573901.

10 Janik J, Chwirot P. Perforated peptic ulcer--time trends and patterns over 20 years. Med Sci Monit 2000;6:369-72.

11 Feliciano DV, Bitondo CG, Burch JM, et al. Emergency management of perforated peptic ulcers in the elderly patient. Am J Surg 1984:148:764-7.

12 Franceschi F, Covino M, Roubaud Baudron C. Review: Helicobacter pylori and extragastric diseases. Helicobacter 2019;24 Suppl 1:e12636

13 Ojetti V, Saviano A, Petruzziello C, et al. 13C urea breath test to identify Helicobacter pylori infection in patients with upper gastrointestinal bleeding admitted to the emergency department. Eur Rev Med Pharmacol Sci 2021;25:804-11.

14 Abrignani MG, Gatta L, Gabrielli D, et al. Gastroprotection in patients on antiplatelet and/or anticoagulant therapy: a position paper of national association of hospital cardiologists (ANMCO) and the Italian association of hospital Gastroenterologists and endoscopists (AIGO). Eur J Intern Med 2021;85:1-13.

15 Yachimski PS, Friedman LS. Gastrointestinal bleeding in the elderly. Nat Clin Pract Gastroenterol Hepatol 2008;5:80-93.

16 Jairath V, Hearnshaw S, Brunskill SJ, et al. Red cell transfusion for the management of upper gastrointestinal haemorrhage. Cochrane Database Syst Rev 2010:CD006613.

17 Hearnshaw SA, Logan RFA, Palmer KR, et al. Outcomes following early red blood cell transfusion in acute upper gastrointestinal bleeding. Aliment Pharmacol Ther 2010;32:215-24.

18 Pacelli F, Rosa F, Marrelli D, et al. Do perioperative blood transfusions influence prognosis of gastric cancer patients? analysis of 927 patients and interactions with splenectomy. Ann Surg Oncol 2011;18:1615-23.

19 Tarasconi A, Coccolini F, Biffl WL, et al. Perforated and bleeding peptic ulcer: WSES guidelines. World J Emerg Surg 2020;15:3.

20 Fransvea P, Costa G, Lepre L, et al. Laparoscopic repair of perforated peptic ulcer in the elderly: an interim analysis of the FRAILESEL Italian multicenter prospective cohort study. Surg Laparosc Endosc Percutan Tech 2020;31:2-7.

21 Ross JT, Matthay MA, Harris HW. Secondary peritonitis: principles of diagnosis and intervention. BMJ 2018;361:k1407.

22 Donovan AJ, Berne TV, Donovan JA. Perforated duodenal ulcer. Arch Surg 1998;133:1166-71.

23 Pacelli F, Rosa F, Marrelli D, et al. Naso-gastric or naso-jejunal decompression after partial distal gastrectomy for gastric cancer. final results of a multicenter prospective randomized trial. Gastric Cancer 2014;17:725-32.

24 Buck DL, Vester-Andersen M, Møller MH. Surgical delay is a critical determinant of survival in perforated peptic ulcer. Br J Surg 2013;100:1045-9.

25 Møller MH, Adamsen S, Thomsen RW, et al. Preoperative prognostic factors for mortality in peptic ulcer perforation: a systematic review. Scand J Gastroenterol 2010:45:785-805.

26 Roviello F, Rossi S, Marrelli D, et al. Perforated gastric carcinoma: a report of 10 cases and review of the literature. World J Surg Oncol 2006;4:19.

27 Quero G, Covino M, Ojetti V, et al. Acute pancreatitis in oldest old: a 10-year retrospective analysis of patients referred to the emergency department of a large tertiary hospital. Eur J Gastroenterol Hepatol 2020;32:159-65.

28 Covino M, Rosa F, Ojetti V, et al. Acute diverticulitis in elderly patients: does age really matter? Dig Dis 2021;39:33-41. 